PROCEEDINGS OF THE

AMERICAN MATHEMATICAL SOCIETY

Volume 140, Number 5, May 2012, Pages 1769-1775

S 0002-9939(2011)11197-3

Article electronically published on August 24, 2011

\title{
RIGIDITY OF STABLE CYLINDERS IN THREE-MANIFOLDS
}

\author{
JOSÉ M. ESPINAR
}

(Communicated by Michael Wolf)

\begin{abstract}
In this paper we show how the existence of a certain stable cylinder determines (locally) the ambient manifold where it is immersed. This cylinder has to verify a bifurcation phenomenon; we make this explicit in the introduction. In particular, the existence of such a stable cylinder implies that the ambient manifold has infinite volume.
\end{abstract}

\section{INTRODUCTION}

A stable compact domain $\Sigma$ on a minimal surface in a Riemannian three-manifold $\mathcal{M}$ is one whose area cannot be decreased up to second order by a variation of the domain leaving the boundary fixed. Stable oriented domains $\Sigma$ are characterized by the stability inequality for normal variations $\psi N[11$

$$
\int_{\Sigma} \psi^{2}|A|^{2}+\int_{\Sigma} \psi^{2} \operatorname{Ric}_{\mathcal{M}}(N, N) \leq \int_{\Sigma}|\nabla \psi|^{2}
$$

for all compactly supported functions $\psi \in H_{0}^{1,2}(\Sigma)$. Here $|A|^{2}$ denotes the square of the length of the second fundamental form of $\Sigma$, $\operatorname{Ric}_{\mathcal{M}}(N, N)$ is the Ricci curvature of $\mathcal{M}$ in the direction of the normal $N$ to $\Sigma$ and $\nabla$ is the gradient w.r.t. the induced metric.

One writes the stability inequality in the form

$$
\left.\frac{d^{2}}{d t^{2}}\right|_{t=0} \operatorname{Area}(\Sigma(t))=-\int_{\Sigma} \psi L \psi \geq 0,
$$

where $L$ is the linearized operator of the mean curvature

$$
L=\Delta+|A|^{2}+\operatorname{Ric}_{\mathcal{M}}
$$

In terms of $L$, stability means that $-L$ is nonnegative; i.e., all its eigenvalues are nonnegative. $\Sigma$ is said to have finite index if $-L$ has only finitely many negative eigenvalues.

From the Gauss equation, one can write the stability operator as $L=\Delta-K+V$, where $\Delta$ and $K$ are the Laplacian and Gauss curvature associated to the metric $g$ respectively and $V:=1 / 2|A|^{2}+S$, where $S$ denotes the scalar curvature associated to the metric $g$.

Received by the editors August 2, 2010 and, in revised form, January 14, 2011.

2010 Mathematics Subject Classification. Primary 53A10; Secondary 53C24, 49Q05.

Key words and phrases. Stable surfaces, bifurcation.

The author is partially supported by the Spanish MEC-FEDER Grant MTM2010-19821 and Regional J. Andalucia Grants P06-FQM-01642 and FQM325.

(C)2011 American Mathematical Society
Reverts to public domain 28 years from publication 
The index form of this kind of operators is

$$
I(f)=\int_{\Sigma}\left\{\|\nabla f\|^{2}-V f^{2}+K f^{2}\right\}
$$

where $\nabla$ and $\|\cdot\|$ are the gradient and norm associated to the metric $g$. Thus, if $\Sigma$ is stable, we have

$$
\int_{\Sigma} f L f=-I(f) \leq 0
$$

or equivalently

$$
\int_{\Sigma} f^{2}\left(1 / 2|A|^{2}+S\right) \leq \int_{\Sigma}\left\{\|\nabla f\|^{2}+K f^{2}\right\}
$$

In a seminar paper [5], D. Fischer-Colbrie and R. Schoen proved:

Theorem A. Let $\mathcal{M}$ be a complete oriented three-manifold of nonnegative scalar curvature. Let $\Sigma$ be an oriented complete stable minimal surface in $\mathcal{M}$. If $\Sigma$ is noncompact, conformally equivalent to the cylinder and the absolute total curvature of $\Sigma$ is finite, then $\Sigma$ is flat and totally geodesic.

They also state [5. Remark 2]: We feel that the assumption of finite total curvature should not be essential in proving that the cylinder is flat and totally geodesic.

Recently, this question was partially answered in [3] under the assumption that the positive part of the Gaussian curvature is integrable, i.e., $K^{+}:=\max \{0, K\} \in$ $L^{1}(\Sigma)$. It was totally answered by M. Reiris [10; he proved:

Theorem B. Let $\mathcal{M}$ be a complete oriented three-manifold of nonnegative scalar curvature. Let $\Sigma$ be an oriented complete stable minimal surface in $\mathcal{M}$ diffeomorphic to the cylinder. Then $\Sigma$ is flat and totally geodesic.

Besides, Bray, Brendle and Neves [1] were able to determine the structure of a three-manifold $\mathcal{M}$ under the assumption of the existence of an area minimizing two-sphere. Specifically, they proved:

Theorem C. Let $\mathcal{M}$ be a compact three-manifold with $\pi_{2}(\mathcal{M}) \neq 0$. Denote by $\mathcal{F}$ the set of all smooth maps $f: \mathbb{S}^{2} \rightarrow \mathcal{M}$ which represent a nontrivial element of $\pi_{2}(\mathcal{M})$. Set

$$
\mathcal{A}(\mathcal{M}):=\inf \left\{\operatorname{area}\left(f\left(\mathbb{S}^{2}\right)\right): f \in \mathcal{F}\right\} .
$$

Then

$$
\mathcal{A}(\mathcal{M}) \inf _{\mathcal{M}} R \leq 8 \pi
$$

where $R$ denotes the scalar curvature of $\mathcal{M}$. Moreover, if the equality holds, then the universal cover of $\mathcal{M}$ is isometric to the standard cylinder $\mathbb{S}^{2} \times \mathbb{R}$ up to scaling.

In this paper, we will go further. We will see how the existence of a stable cylinder verifying a bifurcation phenomenon determines the ambient manifold $\mathcal{M}$. First, let us make clear what we mean by bifurcation phenomenon:

Definition 1.1. We say that a complete minimal surface $\Sigma \subset \mathcal{M}$ bifurcates if there exist $\delta>0$ and a smooth map $u: \Sigma \times(-\delta, \delta) \rightarrow \mathbb{R}$ so that:

- For each $p \in \Sigma$, we have $u(x, 0)=0$ and $\left.\frac{\partial}{\partial t}\right|_{t=0} u(p, t)=1$. Moreover, $u(p, t) \geq 0$ if $t>0$ and $u(p, t) \leq 0$ if $t<0$. 
- For each $t \in(-\delta, \delta)$, the surface

$$
\Sigma_{t}:=\left\{\exp _{p}(u(p, t) N(p)): p \in \Sigma\right\}
$$

is a complete minimal surface. Here, exp denotes the exponential map in $\mathcal{M}$.

Now, we can state

Theorem 1.1. Let $\mathcal{M}$ be a complete oriented Riemannian three-manifold with nonnegative scalar curvature. Assume there exists $\Sigma \subset \mathcal{M}$ a complete stable minimal surface conformally equivalent to a cylinder that bifurcates. Then $\Sigma$ is flat, totally geodesic, and $S$ vanishes along $\Sigma$. Moreover, there exists an open set $\mathcal{U} \subset \mathcal{M}$ so that $\mathcal{U}$ is locally isometric to $\mathcal{C} \times(-\delta, \delta)$, where $\mathcal{C}$ denotes the standard cylinder $\mathbb{S}^{1} \times \mathbb{R}$. Also, if any complete stable cylinder in $\mathcal{M}$ bifurcates for a uniform $\delta>0$, then $\mathcal{M}$ is locally isometric either to $\mathbb{S}^{1} \times \mathbb{R}^{2}$ or $\mathbb{T}^{2} \times \mathbb{R}$ (here $\mathbb{T}^{2}$ is the flat tori).

We should point out that the condition that $\Sigma$ bifurcates is necessary. In fact, one can construct the following example: Let $\mathcal{C}(-l, l)$ be the right cylinder of height $2 l$ and radius 1 endowed with the flat metric. Close it up with two spherical caps $S_{i}, i=1,2$ (one on the top and another on the bottom). Now, smooth the surface $\mathcal{M}^{2}=\mathcal{C}(-l, l) \cup S_{1} \cup S_{2}$ so that it is flat on $\mathcal{C}(-l+\varepsilon, l-\varepsilon)$, for some $\varepsilon>0$, and has nonnegative Gaussian curvature.

Consider the three-manifold $\mathcal{M}^{3}=\mathcal{M}^{2} \times \mathbb{R}$. One can see that, if we take a closed geodesic $\gamma(t) \subset \mathcal{C}(-l+\varepsilon, l-\varepsilon), t \in(-l+\varepsilon, l-\varepsilon)$, the surface $\Sigma(t):=\gamma(t) \times \mathbb{R}$ is a complete stable minimal cylinder in $\mathcal{M}$ that bifurcates, but, when we reach $t=l-\varepsilon$, this property might disappear (it could bifurcate as constant mean curvature surfaces at one side, but not minimal).

One interesting consequence of Theorem 1.1 is the following:

Corollary 1.1. Let $\mathcal{M}$ be a complete oriented Riemannian three-manifold with nonnegative scalar curvature. Assume there exists $\Sigma \subset \mathcal{M}$ a complete stable minimal surface conformally equivalent to a cylinder that bifurcates. Then,

$$
\operatorname{Vol}(\mathcal{M})=+\infty
$$

Actually, the above conclusion (that is, Corollary 1.1) is also valid when the cylinder bifurcates only at one side.

\section{Preliminaries}

We denote by $\mathcal{M}$ a complete connected orientable Riemannian three-manifold, with Riemannian metric $g$. Moreover, throughout this work, we will assume that its scalar curvature is nonnegative, i.e., $S \geq 0$. Also, $\Sigma \subset \mathcal{M}$ will be assumed to be connected and oriented.

We denote by $N$ the unit normal vector field along $\Sigma$. Let $p_{0} \in \Sigma$ be a point of the surface and let $D\left(p_{0}, s\right)$, for $s>0$, denote the geodesic disk centered at $p_{0}$ of radius $s$. We assume that $\overline{D\left(p_{0}, s\right)} \cap \partial \Sigma=\emptyset$. Moreover, let $r$ be the radial distance of a point $p$ in $D\left(p_{0}, s\right)$ to $p_{0}$. We write $D(s)=D\left(p_{0}, s\right)$. 
We also denote

$$
\begin{aligned}
l(s) & =\operatorname{Length}(\partial D(s)), \\
a(s) & =\operatorname{Area}(D(s)), \\
K(s) & =\int_{D(s)} K, \\
\chi(s) & =\text { Euler characteristic of } D(s) .
\end{aligned}
$$

Let $\Sigma \subset \mathcal{M}$ be a stable minimal surface diffeomorphic to the cylinder. Then, from Theorem B [10], $\Sigma$ is flat and totally geodesic. We will give a (more general) proof of this result in the abstract setting of Schrödinger-type operators:

Lemma 2.1. Let $\Sigma$ be a complete Riemannian surface. Let $L=\Delta+V-a K$ be a differential operator on $\Sigma$ acting on compactly supported $f \in H_{0}^{1,2}(\Sigma)$, where $a>1 / 4$ is constant, $V \geq 0$, and $\Delta$ and $K$ are the Laplacian and Gauss curvature associated to the metric $g$ respectively.

Assume that $\Sigma$ is homeomorphic to the cylinder and $-L$ is nonnegative. Then, $V \equiv 0$ and $K \equiv 0$. Therefore,

$$
\operatorname{Ker} L:=\{1\}
$$

i.e., its kernel is the constant functions. Here, $L$ denotes the Jacobi operator.

Proof. Set $b \geq 1$ and let us consider the radial function

$$
f(r):=\left\{\begin{array}{cc}
(1-r / s)^{b}, & r \leq s \\
0, & r>s
\end{array}\right.
$$

where $r$ denotes the radial distance from a point $p_{0} \in \Sigma$. Then, from [3, Lemma 3.1] (see also 9]), we have

$$
\int_{D(s)}(1-r / s)^{2 b} V \leq 2 a \pi G(s)+\frac{b(b(1-4 a)+2 a)}{s^{2}} \int_{0}^{s}(1-r / s)^{2 b-2} l(r),
$$

where

$$
G(s):=-\int_{0}^{s}\left(f(r)^{2}\right)^{\prime} \chi(r) .
$$

Therefore, since $a>1 / 4$, we can find $b \geq 1$ so that $b(1-4 a)+2 a \leq 0$. So

$$
\int_{D(s)}(1-r / s)^{2 b} V \leq 2 a \pi G(s) .
$$

- Step 1: $V$ vanishes identically on $\Sigma$.

Suppose there exists a point $p_{0} \in \Sigma$ so that $V\left(p_{0}\right)>0$. From now on, we fix the point $p_{0}$. Then, there exists $\epsilon>0$ so that $V(q) \geq \delta$ for all $q \in D(\epsilon)=D\left(p_{0}, \epsilon\right)$. Since $\Sigma$ is topologically a cylinder, there exists $s_{0}>0$ so that for all $s>s_{0}$ we have $\chi(s) \leq 0$ (see [2, Lemma 1.4]).

Now, from the above considerations, there exists $\beta>0$ so that

$$
0<\beta \leq 2 a \pi G(s) .
$$


But, following [3], we can see that

$$
\begin{gathered}
G(s)=-\int_{0}^{s}\left(f(r)^{2}\right)^{\prime} \chi(r)=-\int_{0}^{s_{0}}\left(f(r)^{2}\right)^{\prime} \chi(r)-\int_{s_{0}}^{s}\left(f(r)^{2}\right)^{\prime} \chi(r) \\
\leq-\int_{0}^{s_{0}}\left(f(r)^{2}\right)^{\prime}=-\left(f\left(s_{0}\right)^{2}-f(0)^{2}\right)=-f\left(s_{0}\right)^{2}+1 \\
=-\left(1-s_{0} / s\right)^{2 b}+1 \\
\text { since }-\int_{s_{0}}^{s}\left(f(r)^{2}\right)^{\prime} \chi(r) \geq 0 . \text { Therefore, } \\
\quad G(s) \leq 1-\left(1-s_{0} / s\right)^{2 b} \rightarrow 0, \text { as } s \rightarrow+\infty
\end{gathered}
$$

which is a contradiction. Thus, $V$ vanishes identically along $\Sigma$.

- Step 2: $K$ vanishes identically on $\Sigma$. In particular, $\Sigma$ is parabolic.

First, note that $L:=\Delta-a K$. From [4, there is a smooth positive function $u$ on $\Sigma$ such that $L u=0$. Set $\alpha:=1 / a$. Then, from 9] (following ideas of [4), the conformal metric $\tilde{d s}^{2}:=u^{2 \alpha} d s^{2}$, where $d s^{2}$ is the metric on $\Sigma$, is complete and its Gaussian curvature $\tilde{K}$ is nonnegative; i.e., $\tilde{K} \geq 0$.

On the one hand, the respective Gaussian curvatures are related by

$$
\alpha \Delta \ln u=K-\tilde{K} u^{2 \alpha} .
$$

On the other hand, since $\Sigma$ is topologically a cylinder, the Cohn-Vossen inequality says

$$
\int_{\Sigma} \tilde{K} \leq 0
$$

that is, $\tilde{K}$ vanishes identically.

Thus, $K=\alpha \Delta \ln u$. From this last equation, we get

$$
a K=\frac{1}{u} \Delta u-\frac{|\nabla u|^{2}}{u^{2}}
$$

that is,

$$
\frac{|\nabla u|^{2}}{u}=\Delta u-a K u=0
$$

This last equation implies that $u$ is constant, and since $u$ satisfies $L u=0$, we have that $K$ vanishes identically on $\Sigma$. In particular, $\Sigma$ is parabolic (see [6. Lemma 5]).

This implies that the Jacobi operator becomes $L:=\Delta$, and so the constant functions are in the kernel. But, since $\Sigma$ is parabolic, such a kernel has dimension one (see [8]); therefore

$$
\operatorname{Ker} L:=\{1\} .
$$

Let $\mathcal{C}:=\mathbb{S}^{1} \times \mathbb{R}$ be the flat cylinder. Then we can parametrize $\Sigma$ as the isometric immersion $\psi_{0}: \mathcal{C} \rightarrow \mathcal{M}$ where $\Sigma:=\psi_{0}(\mathcal{C})$. Also, let $N_{0}: \mathcal{C} \rightarrow N \Sigma$ be the unit normal vector field along $\Sigma$.

Assume $\Sigma$ bifurcates (see Definition 1.1). Then there exist $\delta>0$ and a smooth map $u: \mathcal{C} \times(-\delta, \delta) \rightarrow \mathbb{R}$ so that the surface $\Sigma_{t}:=\psi_{t}(\mathcal{C}), \psi_{t}: \mathcal{C} \rightarrow \mathcal{M}$ where

$$
\psi_{t}(p):=\exp _{\psi_{0}(p)}\left(u(p, t) N_{0}(p)\right), p \in \mathcal{C}
$$

is a complete minimal surface. 
For each $t \in(-\delta, \delta)$, the lapse function $\rho_{t}: \Sigma \rightarrow \mathbb{R}$ is defined by

$$
\rho_{t}(p)=g\left(N_{t}(p), \frac{\partial}{\partial t} \psi_{t}(p)\right) .
$$

Clearly, $\rho_{0}(p)=1$ for all $p \in \mathcal{C}$. Also, the lapse function satisfies the Jacobi equation

$$
\Delta_{t} \rho_{t}+\left(\operatorname{Ric}\left(N_{t}\right)+\left|A_{t}\right|^{2}\right) \rho_{t}=0,
$$

since $\psi_{t}(\mathcal{C})$ is minimal for all $|t|<\delta$.

Lemma 2.2. There exists $0<\delta^{\prime}<\delta$ such that $\Sigma_{t}$ is a stable minimal surface for each $t \in(-\delta, \delta)$. Thus, $\Sigma_{t}$ is flat, totally geodesic, and $S$ vanishes along $\Sigma_{t}$ for each $t \in(-\delta, \delta)$.

Proof. First, note that the lapse function is not negative for all $|t|<\delta$ and therefore, by (2.1) and the Maximum Principle, either $\rho_{t}$ vanishes identically or $\rho_{t}>0$ for each $|t|<\delta$.

So, since

$$
\rho_{t} \rightarrow \rho_{0} \equiv 1 \text { as } t \rightarrow 0
$$

we can find a uniform constant $0<\delta^{\prime}<\delta$ such that $\rho_{t}>0$ for all $|t| \leq \delta^{\prime}$.

Therefore, $\rho_{t},|t| \leq \delta^{\prime}$, is a positive function solving the Jacobi equation. This implies that $\Sigma_{t}$ is stable for all $|t| \leq \delta^{\prime}$ (see [4]).

The last assertion follows from Lemma 2.1 and $\Sigma_{t}$ is stable.

\section{Proof of Theorem 1.1}

From Definition 1.1 and Lemma 2.2, there exists $\delta>0$ so that $\Sigma_{t}$ is a complete minimal stable surface, which is flat, totally geodesic and $S=0$ along $\Sigma_{t}$, for each $|t|<\delta$.

Now, we follow ideas of [1]. Since $\operatorname{Ric}\left(N_{t}\right)+\left|A_{t}\right|^{2} \equiv 0$ and $H(t)=0$ for each $|t|<\delta$, from (2.1) and $\Sigma_{t}$ being parabolic, we obtain that $\rho_{t}$ is constant. Thus, since $\Sigma_{t}$ is totally geodesic,

$$
\begin{array}{rlc}
Y: \mathcal{C} \times(-\delta, \delta) & \rightarrow & \mathcal{M} \\
(p, t) & \rightarrow Y(p, t):=N_{t}(p)
\end{array}
$$

is parallel. Also, the flow of $N_{t}$ is a unit speed geodesic flow (see [7]). Moreover, the map

$$
\begin{aligned}
& \Phi: \Sigma \times(-\delta, \delta) \rightarrow \quad \rightarrow \quad \mathcal{M} \\
& (p, t) \rightarrow \Phi(p, t):=\exp _{\psi_{0}(p)}(t N(p))
\end{aligned}
$$

is a local isometry onto $\mathcal{U}=\bigcup_{|t|<\delta} \Sigma_{t}$. Therefore, $\Phi$ is a diffeomorphism onto $\mathcal{U}$, which implies that $Y: \mathcal{C} \times(-\delta, \delta) \rightarrow \mathcal{U}$ is a globally defined unit Killing vector field. This implies that $\mathcal{U}$ is locally isometric to $\mathcal{C} \times(-\delta, \delta)$.

Now, assume that any stable minimal complete cylinder bifurcates for a uniform $\delta>0$. Then, we can start with a complete stable minimal cylinder $\Sigma_{0}$ that bifurcates, and then by the above considerations, $\Sigma_{t}$, for each $|t|<\delta$, is complete, flat, totally geodesic and $S$ vanishes along $\Sigma_{t}$. Moreover, $\Sigma_{t}$ is strongly stable for each $|t|<\delta$. Note that $\Sigma_{\delta}$ is a strongly stable minimal surfaces conformally equivalent to a cylinder. Since it is the limit of strongly stable minimal surfaces $\Sigma_{t}$ which are flat and totally geodesic, then $\Sigma_{\delta}$ is totally geodesic, flat and $S=0$ along $\Sigma_{\delta}$. Therefore, by Definition 1.1 and Lemma 2.2 there exists $\delta>0$ so that $\Sigma_{t},-\delta<t<2 \delta$, 
is flat, totally geodesic and $S$ vanishes along $\Sigma_{t}$. Continuing this argument, $\Sigma_{t}$ is flat, totally geodesic and $S$ vanishes along $\Sigma_{t}$ for each $t \in \mathbb{I}$, where $\mathbb{I}=\mathbb{R}$ or $\mathbb{I}=\mathbb{S}^{1}$.

As we did above, since $\operatorname{Ric}\left(N_{t}\right)+\left|A_{t}\right|^{2} \equiv 0$ and $H(t)=0$ for each $t \in \mathbb{I}$, from (2.1) and $\Sigma_{t}$ being parabolic, we obtain that $\rho_{t}$ is constant. Thus, since $\Sigma_{t}$ is totally geodesic,

$$
\begin{array}{rlc}
Y: \mathcal{C} \times \mathbb{I} & \rightarrow & \mathcal{M} \\
(p, t) & \rightarrow & \rightarrow(p, t):=N_{t}(p)
\end{array}
$$

is parallel, where $\mathbb{I}=\mathbb{R}$ or $\mathbb{I}=\mathbb{S}^{1}$. Also, the flow of $N_{t}$ is a unit speed geodesic flow (see [7). Moreover, the map

$$
\begin{aligned}
& \Phi: \Sigma \times \mathbb{I} \rightarrow \quad \mathcal{M} \\
& (p, t) \rightarrow \Phi(p, t):=\exp _{\psi_{0}(p)}(t N(p))
\end{aligned}
$$

is a local isometry, which implies that it is a covering map. Therefore, $\Phi$ is a diffeomorphism, which implies that $Y: \mathcal{C} \times \mathbb{I} \rightarrow \mathcal{M}$ is a globally defined unit Killing vector field. This implies that $\mathcal{M}$ is locally isometric either to $\mathbb{S}^{1} \times \mathbb{R}^{2}$ or $\mathbb{T}^{2} \times \mathbb{R}$ (here $\mathbb{T}^{2}$ denotes the flat tori).

\section{REFERENCES}

[1] H. Bray, S. Brendle and A. Neves: Rigidity of area-minimizing two-spheres in three-manifolds. Comm. Anal. Geom. 18 (2010), no. 4, 821-830. MR.2765731

[2] P. Castillon: An inverse spectral problem on surfaces, Comment. Math. Helv. 81 (2006), no. 2, 271-286. MR2225628(2007b:58042)

[3] J.M. Espinar and H. Rosenberg: A Colding-Minicozzi stability inequality and its applications. Trans. A.M.S. 363 (2011), 2447-2465. MR2763722

[4] D. Fischer-Colbrie: On complete minimal surfaces with finite Morse index in three manifolds, Invent. Math. 82 (1985), 121-132. MR808112 (87b:53090)

[5] D. Fischer-Colbrie and R. Schoen: The structure of complete stable minimal surfaces in 3-manifolds of nonnegative scalar curvature, Comm. Pure Applied Math. 33 (1980), 199-211. MR562550(81i:53044)

[6] T. Klotz and R. Osserman: Complete surfaces in $E^{3}$ with constant mean curvature, Comment. Math. Helv. 41 (1966-67), 313-318. MR0211332 (35:2213)

[7] S. Montiel: Unicity of constant mean curvature hypersurfaces in some Riemannian manifolds, Indiana Univ. Math. J. 48 (1999), 711-748. MR1722814(2001f:53131)

[8] J. M. Manzano, J. Pérez and M. M. Rodríguez: Parabolic stable surfaces with constant mean curvature. To appear in Calc. Var. Partial Differential Equations.

[9] W. Meeks, J. Pérez and A. Ros: Stable constant mean curvature surfaces, Handbook of Geometric Analysis, volume 1 (2008), pages 301-380. International Press, edited by Lizhen Ji, Peter Li, Richard Schoen and Leon Simon. MR2483369 (2009k:53016)

[10] M. Reiris: Geometric relations of stable minimal surfaces and applications. Preprint.

[11] R. Schoen and S.T. Yau: Harmonic maps and the topology of stable hypersurfaces and manifolds of nonnegative Ricci curvature, Comm. Math. Helv. 39 (1976), 333-341. MR0438388 $(55: 11302)$

Departamento de Geometría y Topología, Universidad de Granada, 18071 Granada, SPAIN

E-mail address: jespinar@ugr.es 